\title{
Antenna Design through Space Mapping Optimization
}

\author{
Jiang Zhu, John W. Bandler, Fellow, IEEE, Natalia K. Nikolova, Senior Member, IEEE, \\ and Slawomir Koziel, Member, IEEE
}

McMaster University, Hamilton, ON, Canada L8S 4K1, www.sos.mcmaster.ca

\begin{abstract}
For the first time, we apply space mapping to antenna design. We exploit a coarse-mesh method-of-moments (MoM) solver as the coarse model and align it with the fine-mesh MoM solution through implicit space mapping and output space mapping. A novel local meshing method avoids inconsistencies in the coarse model. Our new user-friendly SMF system optimizes the impedance of a patch antenna in four iterations. In a double annular ring antenna the finite ground size effect for the MoM is efficiently solved and the design specification is satisfied after only three iterations.
\end{abstract}

Index Terms-Antenna design, CAD, EM optimization, method of moments, surrogate modeling, space mapping.

\section{INTRODUCTION}

The method of moments (MoM) is one of the most frequently used numerical techniques for analysis of planar structures. An accurate MoM simulation is cpu intensive. This cost may be prohibitive for complex design problems. Alternatively, a typical coarse mesh MoM simulation is fast, but poor in accuracy.

The space mapping (SM) technique takes advantage of the efficiency of a coarse mesh MoM simulation and the accuracy of the fine mesh simulation. SM aligns coarse models with fine models [1]-[3]. Here, the fine model is the fine mesh MoM solution. The coarse model is the coarse mesh solution.

For the first time, we apply the SM technique to antenna design. Both fine and coarse models are defined within the commercial EM solver FEKO, which is used primarily for antenna design based on the MoM [4]. We propose a new meshing method: the topology of the mesh is preserved throughout the optimization which makes the coarse-model response in the design parameter space smooth and consistent.

In the parameter extraction (PE) process, we implement implicit SM (ISM) and output SM (OSM) sequentially [2], [3]. In a preliminary PE, we roughly align the coarse model with the fine model through ISM. Then OSM aims at locally matching the surrogate with the fine model.

This work was supported in part by the Natural Sciences and Engineering Research Council of Canada under Grants OGP0007239 and STGP269760, and by Bandler Corporation.

The authors are with the Simulation Optimization Systems and Computational Electromagnetics Research Laboratories, Department of Electrical and Computer Engineering, McMaster University, Hamilton, ON, Canada L8S 4K1.

J.W. Bandler is also with Bandler Corporation, P.O. Box 8083, Dundas, ON, Canada L9H 5 E7.
Our new SMF system [5] implements our approach. SMF is a GUI-oriented Matlab space-mapping nonlinear optimization software package with sockets to popular EM simulators, including FEKO, Sonnet $\boldsymbol{e} \boldsymbol{m}$ and Agilent ADS.

We consider a patch antenna and a double annular ring antenna. In the first example, we optimize impedance at a single frequency, and then discuss the coarseness in the coarse model and its effect on the SM performance. In the second example, we exploit the cpu-intensive surface equivalence principle (SEP) as a fine model and the special Green's function with a coarse mesh as a coarse model. The $S$ parameter response is optimized in 3 iterations.

\section{Mesh Convergence And Meshing Method}

The mesh convergence needs to be checked to get an accurate simulation result. This is done by refining the mesh from one simulation to the next, and keeping all other parameters the same. If the results are significantly different, the surfaces are not adequately discretized and we need to refine the mesh [6].

The coarse-mesh coarse model does not need to achieve mesh convergence. Consequently, the variation of the mesh number and topology due to the variation of geometrical design parameters during optimization leads to inconsistent results. To overcome this problem, we force the mesh number and topology to remain unchanged during optimization. This is done by local meshing in FEKO.

In the fine model, where mesh convergence is satisfied, we use global meshing. We define the mesh density by the number of meshes per wavelength.

\section{SM-BASEd SurRogate Models}

We are concerned with a class of optimization algorithms that exploit surrogate models [7]. Let $\boldsymbol{R}_{f}: X_{f} \rightarrow R^{m}$ denote the response vector of the so-called fine model of a given object, where $X_{f} \subseteq R^{n}$. Our goal is to solve

$$
\boldsymbol{x}_{f}^{*}=\arg \min _{\boldsymbol{x}_{f} \in X_{f}} U\left(\boldsymbol{R}_{f}\left(\boldsymbol{x}_{f}\right)\right)
$$

where $U$ is a suitable objective function.

SM assumes the existence of a less accurate but much faster coarse model. Let $\boldsymbol{R}_{c}: X_{c} \times X_{p} \rightarrow R^{m}$ denote the response vectors of the coarse model, where $X_{c} \subseteq R^{n}$ is the design 
variable domain (we assume here that $X_{c} \subseteq X_{f}$ ) and $X_{p} \subseteq R^{q}$ is the domain of auxiliary (preassigned) coarse model parameters. Typical preassigned parameters $\boldsymbol{x}_{p}$ are the dielectric constant and the height of a dielectric layer. By $\boldsymbol{x}_{c}^{*}$ we denote the optimal solution of the coarse model, i.e.,

$$
\boldsymbol{x}_{c}^{*} \triangleq \arg \min _{\boldsymbol{x} \in X_{c}} U\left(\boldsymbol{R}_{c}\left(\boldsymbol{x}, \boldsymbol{x}_{p}^{(0)}\right)\right)
$$

where $\boldsymbol{x}_{p}^{(0)}$ denotes the initial preassigned parameter values.

We consider the fine model to be expensive to compute and solving (1) by direct optimization to be impractical. Instead, we use surrogate models, i.e., models that are not as accurate as the fine model but are computationally cheap, hence suitable for iterative optimization. We consider an optimization algorithm that generates a sequence of points $\boldsymbol{x}_{f}^{(i)} \in X_{f}, i=1,2, \ldots$, so that

$$
\boldsymbol{x}_{f}^{(i+1)}=\arg \min _{x \in X_{c}} U\left(\boldsymbol{R}_{s}^{(i)}(\boldsymbol{x})\right) .
$$

Here, $\boldsymbol{R}_{s}^{(i)}: X_{c} \rightarrow R^{m}$ is the surrogate model at iteration $i$, which uses the coarse model and the fine model data.

In this work, we use a surrogate model based on implicit SM [2] and output SM [3]. The surrogate model at iteration $i$ is defined as

$$
R_{s}^{(i)}(\boldsymbol{x})=R_{c}\left(\boldsymbol{x}, x_{p}^{(i)}\right)+\Delta R^{(i)} .
$$

where

$$
\boldsymbol{x}_{p}^{(i)}=\arg \min _{\boldsymbol{x}_{p} \in X_{p}}\left\|\boldsymbol{R}_{f}\left(\boldsymbol{x}_{f}^{(i)}\right)-\boldsymbol{R}_{c}\left(\boldsymbol{x}_{f}^{(i)}, \boldsymbol{x}_{p}\right)\right\|
$$

and

$$
\Delta \boldsymbol{R}^{(i)}=R_{f}\left(x_{f}^{(i)}\right)-R_{c}\left(x_{f}^{(i)}, x_{p}^{(i)}\right) .
$$

Both implicit and output SM aim at reducing the misalignment between the fine model and the current surrogate, however ISM exploits the physics-based similarity of the models, while the OSM ensures perfect local alignment between the models at the current iteration point. As follows from equations (4)-(6), we implement ISM and OSM sequentially. This is illustrated in Fig.1.
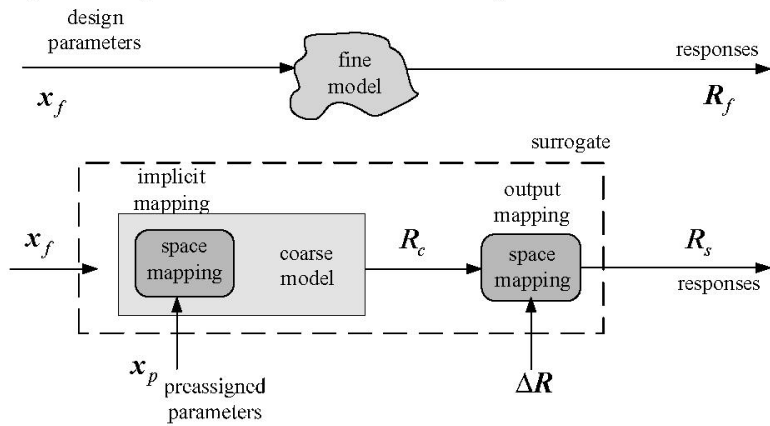

Fig. 1. Demonstration of our approach to implicit and output SM.

We implement the SMF system [5] as follows.
Step 1 Choose a proper coarse-mesh coarse model as well as preassigned parameters. Set $i=0$.

Step 2 Solve (2) to find the surrogate optimal solution $\boldsymbol{x}_{c}^{*}$ and let $\boldsymbol{x}_{f}^{(0)}=\boldsymbol{x}_{c}^{*}$.

Step 3 Evaluate the fine model to find $\boldsymbol{R}_{f}\left(\boldsymbol{x}_{f}^{(i)}\right)$.

Step 4 Update the surrogate model $\boldsymbol{R}_{s}^{(i)}$ according to (4)-(6).

Step 5 Solve (3) and obtain $\boldsymbol{x}_{f}^{(i+1)}$.

Step 6 If the termination condition is satisfied (convergence achieved or the design specification satisfied), stop; otherwise, set $i=i+1$ and go to Step 3 .

\section{EXAMPLES}

\section{A. Patch Antenna}

This patch antenna is printed on a substrate with relative dielectric constant $\varepsilon_{r}=2.32$ and height $h=1.59 \mathrm{~mm}$. The design parameters are the patch length and width, i.e., $\boldsymbol{x}_{f}=[L W]^{T}$. The objective is to obtain $50 \Omega$ input impedance at $2 \mathrm{GHz}$. The objective function is $\left|Z_{\text {in }}-50\right|$.

In the fine model, the global mesh density is 30 meshes per wavelength. In the coarse model, the mesh number and topology are fixed through local meshing. We choose three mesh edges along $L$ and seven along $W$. See Fig. 2. Our selected preassigned SM parameter is $\boldsymbol{x}=\varepsilon_{r}$. In the PE, we match the complex $S_{11}$ instead of the input impedance.

The initial design point is the coarse model optimal solution $x_{f}^{(0)}=\left[\begin{array}{ll}47.1285 & 100.470\end{array}\right] \mathrm{mm}$. The SMF system requires 5 iterations ( 6 fine model simulations). Fig. 3 shows the reduction of the objective function versus the number of the iterations. The final design is $x_{f}^{*}=x_{f}^{(4)}=\left[\begin{array}{ll}46.7294 & 99.6875\end{array}\right]$ $\mathrm{mm}$. Table I shows the optimization results for the design parameters, the objective function value, the preassigned parameter and the output SM parameter at each iteration. Computation time is 341 seconds, compared with 2816 seconds for direct fine model optimization.

Table II discusses the effect on the SM performance of the coarseness of the coarse model for the patch antenna. The algorithm does not converge for 24 meshes. With increase of the number of meshes, the function evaluation time increases while SM iterations decrease. We have the best SM performance in terms of total time cost for 100 meshes, which requires only 341 seconds.

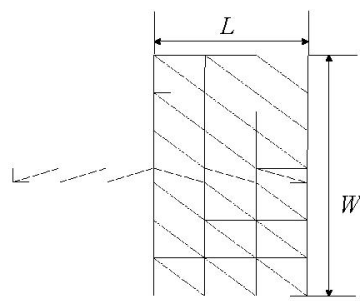

(a)

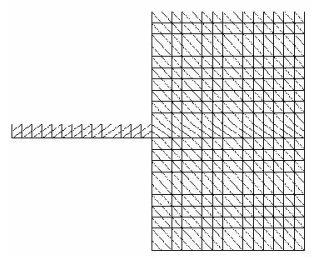

(b)
Fig. 2. Demonstration of coarse model and fine model. (a) Coarse model with three mesh edges along $L$ and seven mesh edges along $W$. (b) Fine model with global mesh density of 30 meshes per wavelength 


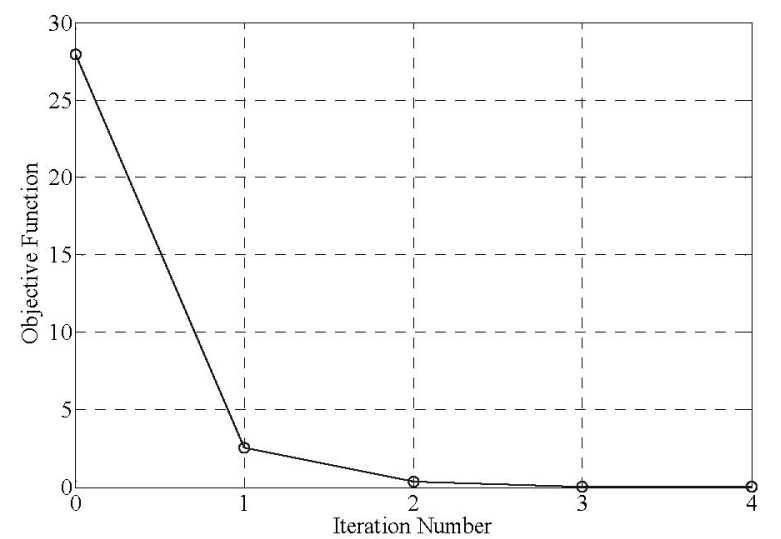

Fig. 3. Objective function value versus iteration number in the microstrip patch antenna example.

TABLE I

Optimization Results For the PATCh Antenna Example

\begin{tabular}{ccccc}
\hline \hline Iteration & $\boldsymbol{x}_{f}(\mathrm{~mm})$ & $\varepsilon_{r}$ & $\Delta R$ & $\left|Z_{\text {in }}-50\right|$ \\
\hline 0 & {$\left[\begin{array}{l}47.1285 \\
100.470\end{array}\right]$} & 2.3200 & 0.0000 & 27.941 \\
1 & {$\left[\begin{array}{l}46.77743 \\
99.6922\end{array}\right]$} & 2.3621 & $0.0082-0.0461 i$ & 2.5616 \\
2 & {$\left[\begin{array}{l}46.7268 \\
99.6960\end{array}\right]$} & 2.3589 & $0.0145+0.0056 i$ & 0.35956 \\
3 & {$\left[\begin{array}{l}46.7294 \\
99.6883\end{array}\right]$} & 2.3590 & $0.0118+0.0054 i$ & $2.4437 \times 10^{-2}$ \\
4 & {$\left[\begin{array}{l}46.7294 \\
99.6875\end{array}\right]$} & 2.3589 & $0.0115+0.0059 i$ & $1.1234 \times 10^{-2}$ \\
\hline \hline
\end{tabular}

TABLE II

The Effect of Local Meshing on SM Performante FOR THE PATCH ANTENNA

\begin{tabular}{|c|c|c|c|c|c|}
\hline \multicolumn{4}{|c|}{ Local meshing in the coarse model } & \multicolumn{2}{|c|}{ SM performance } \\
\hline \multicolumn{2}{|c|}{ Mesh number along } & \multirow{2}{*}{$\begin{array}{c}\text { Total mesh } \\
\text { number }\end{array}$} & \multirow{2}{*}{$\begin{array}{l}\text { Function } \\
\text { evaluation } \\
\text { time (s) }\end{array}$} & \multirow{2}{*}{$\begin{array}{c}\text { Iteration } \\
\text { number }\end{array}$} & \multirow{2}{*}{$\begin{array}{c}\text { Total time } \\
\text { (s) }\end{array}$} \\
\hline$L$ & $W$ & & & & \\
\hline 2 & 5 & 24 & 0.109 & \multicolumn{2}{|c|}{ Not convergent } \\
\hline 3 & 7 & 48 & 0.219 & 6 & 364 \\
\hline 5 & 9 & 100 & 0.438 & 4 & 341 \\
\hline 9 & 20 & 400 & 4.063 & 4 & 1604 \\
\hline 11 & 22 & 528 & 8.375 & 3 & 2695 \\
\hline \multicolumn{4}{|c|}{ Global meshing in the fine model } & \multicolumn{2}{|c|}{ Direct optimization } \\
\hline \multicolumn{2}{|c|}{ Mesh density $=30$} & $1032^{*}$ & $33.313^{*}$ & 0 & 2816 \\
\hline
\end{tabular}

* The number of meshes and function evaluation time in the fine model is measured at the starting point $[L W]=\left[\begin{array}{ll}55 & 85\end{array}\right] \mathrm{mm}$

\section{B. Double Annular Ring Antenna}

We consider the stacked probe-fed printed annular ring antenna of [8] which is shown in Fig. 4. The antenna is printed on a PCB with $\varepsilon_{r 1}=2.2, d_{1}=6.096 \mathrm{~mm}$ for the lower substrate and $\varepsilon_{r 2}=1.07, d_{2}=8.0 \mathrm{~mm}$ for the upper substrate. The dielectric loss tangent is 0.001 for both layers.

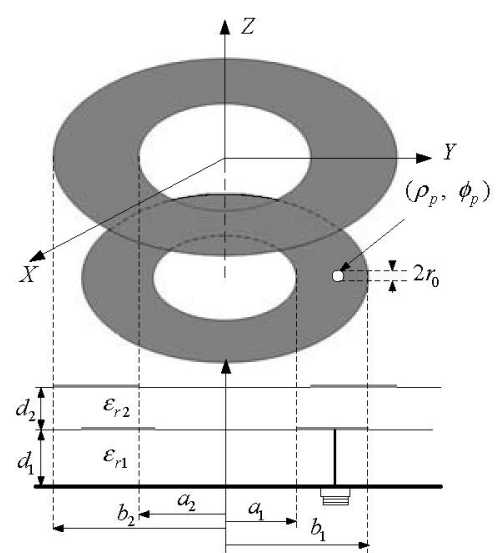

Fig. 4. Geometry of a stacked probe-fed printed double annular ring antenna example.

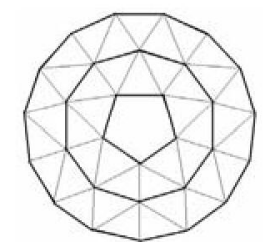

Fig. 5. Demonstration of local meshing of the annular ring in the coarse-mesh coarse model for a stacked probe-fed printed double ring antenna example.

The finite ground size is $100 \times 100 \mathrm{~mm}$. The radius of the feed pin is $r_{0}=0.325 \mathrm{~mm}$. Design variables are the outer and inner radius of each ring and the feed position, namely, $\left[\begin{array}{lllll}a_{1} & a_{2} & b_{1} & b_{2} & \rho_{p}\end{array}\right]^{T}$. The design specification is

$$
\left|S_{11}\right| \leq-10 \mathrm{~dB} \text { for } 1.75 \mathrm{GHz} \leq \omega \leq 2.15 \mathrm{GHz} \text {. }
$$

In an MoM solver like FEKO, special Green's functions are usually implemented to model multi-layer substrates, where the ground and the substrate are assumed infinite in extent. It is computer-resource efficient, since only the finite metallic surfaces are discretized. However, in many microwave and $\mathrm{RF}$ applications, the infinite ground plane assumption is not acceptable for accurate simulations. The ground size has a strong effect on the performance of microstrip antennas.

The surface equivalence principle (SEP) addresses this problem. The surface of a dielectric is discretized for the electric and magnetic currents on the surface. All sides of a dielectric have to be meshed, making a closed solid. In this approach, the memory requirement is four times what it would be if the same structure was metallic [9].

We choose the SEP model as the fine model and the special Green's function, which does not consider the finite ground size effect, as the coarse model. To further reduce the simulation time in the coarse model, we apply a coarser mesh by local meshing. As shown in Fig. 5, the number of mesh edges along the three loops (thick lines) is topologically fixed at 5, 10 and 15, respectively, regardless of the variation in the design parameter values. The detailed fine and coarse models are shown in Table III. 
TABLE III

Fine Model and Coarse Model in Double Annular RING ANTENNA

\begin{tabular}{ccccc}
\hline \hline $\begin{array}{c}\text { Model } \\
\text { type }\end{array}$ & Technique & Meshing method & $\begin{array}{c}\text { Mesh } \\
\text { number }\end{array}$ & $\begin{array}{c}\text { Frequency } \\
\text { sweep time }\end{array}$ \\
\hline $\begin{array}{c}\text { Coarse } \\
\text { model }\end{array}$ & $\begin{array}{c}\text { Special Green's } \\
\text { function + } \\
\text { coarse mesh }\end{array}$ & Local meshing & 83 & 8.721 seconds \\
\hline $\begin{array}{c}\text { Fine } \\
\text { model }\end{array}$ & SEP & $\begin{array}{c}\text { Global meshing } \\
\text { density }=20\end{array}$ & $2661^{*}$ & $\begin{array}{c}1 \text { hour and 18 } \\
\text { minutes }\end{array}$ \\
\hline
\end{tabular}

* Number of meshes and time cost in fine the model are measured at the initial point

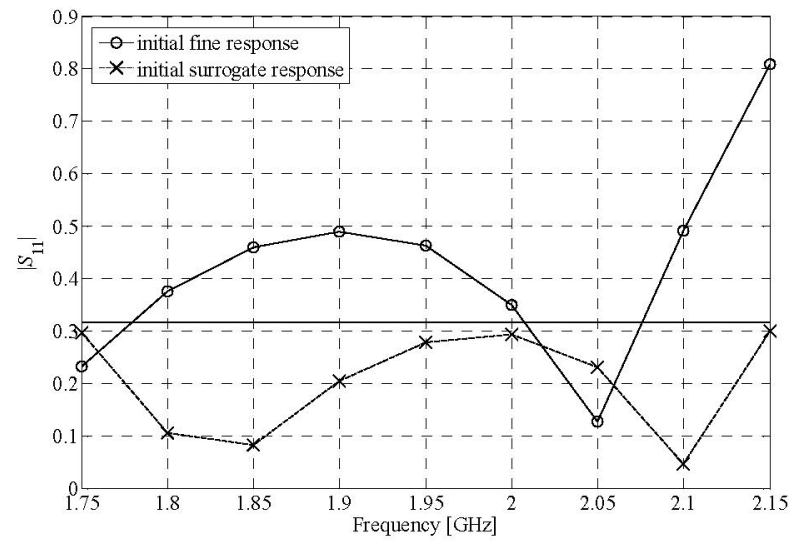

Fig. 6. Initial fine and surrogate responses corresponding to the coarse model optimal solution for the double annular ring antenna.

The SMF system needs 3 iterations (4 fine simulations) to satisfy the specifications, although the coarse model initially exhibits a poor response [see Fig. 6]. The total time taken is 5 hours 58 minutes (a single fine model simulation requires 1 hour 18 minutes). Fig. 7 depicts the fine and surrogate model responses at the final design. Good alignment is achieved with three PEs. Table IV shows the initial and final design.

\section{CONCLUSIONS}

We have presented an effective space-mapping technique for antenna optimization based on a coarse model which exploits a coarse non-convergent mesh of fixed topology. Both coarse and fine models are implemented by one MoM solver. A separate coarse model is not required. We apply our novel SMF system to patch antenna optimization. In the double annular ring example, our SM technique provides an efficient way to address the finite ground size problem. Although we demonstrate our approach through antenna design, it is also applicable to other planar structures.

\section{ACKNOWLEDGEMENT}

The authors thank Dr. C.J. Reddy and his FEKO team for useful discussions and making FEKO available. They thank

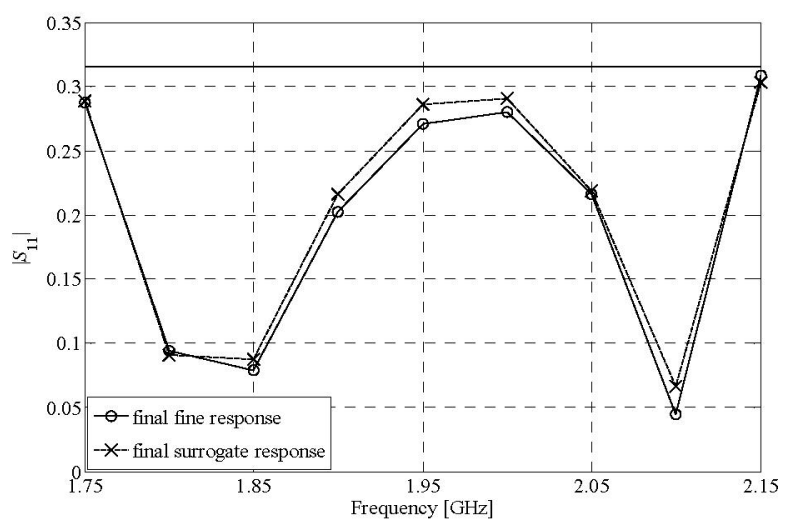

Fig. 7. Final fine and surrogate responses for the double annular ring antenna example.

TABLE IV

InITIAL AND FINAL Design of tHe DOUble ANNULAR Ring ANTENNA

\begin{tabular}{ccc}
\hline $\begin{array}{c}\text { Design } \\
\text { parameters }\end{array}$ & $\begin{array}{c}\text { Initial design } \\
(\mathrm{mm})\end{array}$ & $\begin{array}{c}\text { Final design } \\
(\mathrm{mm})\end{array}$ \\
\hline$a_{1}$ & 9.2277 & 10.6735 \\
$a_{2}$ & 8.7224 & 7.8088 \\
$b_{1}$ & 30.7230 & 28.4621 \\
$b_{2}$ & 34.1266 & 32.5043 \\
$\rho_{p}$ & 18.2107 & 19.6817 \\
\hline \hline
\end{tabular}

Dr. A.S. Mohamed and W. Yu of McMaster University for insightful comments.

\section{REFERENCES}

[1] J.W. Bandler, R.M. Bienacki, S.H. Chen, P.A. Grobelny and R.H. Hemmers, "Space mapping technique for electromagnetic optimization," IEEE Trans. Microwave Theory Tech., vol. 42, pp. 2536-2544, Dec. 1994.

[2] J.W. Bandler, Q.S. Cheng, N.K. Nikolova and M. A. Ismail, "Implicit space mapping optimization exploiting preassigned parameters," IEEE Trans. Microwave Theory Tech., vol. 52, pp. 378-385, Jan. 2004.

[3] J.W. Bandler, Q.S. Cheng, D. Gebre-Mariam, K. Madsen, F. Pedersen and J. Søndergaard, "EM-based surrogate modeling and design exploiting implicit, frequency and output space mappings," in IEEE MTT-S IMS Dig., June 2003, pp.1003-1006.

[4] FEKO $^{\circledR}$ User's Manual, Suite 4.2, June 2004, EM Software \& Systems-S.A. (Pty) Ltd, 32 Techno Lane, Technopark, Stellenbosch, 7600, South Africa, http://www.feko.info.

[5] SMF, Bandler Corporation, P.O. Box 8083, Dundas, ON, Canada L9H 5E7, 2006.

[6] "Mesh refinement," FEKO Quarterly December 2004.

[7] S. Koziel, J.W. Bandler and K. Madsen, "Towards a rigorous formulation of the space mapping technique for engineering design," Proc. ISCAS, Kobe, Japan, June 2005, pp. 5605-5608.

[8] D.M. Kotokoff, J.T. Aberle and R.B. Waterhouse, "Rigorous analysis of probe-fed printed annular ring antennas," IEEE Trans. Antennas Propagat, vol. 47, pp. 384-388, Feb. 1999.

[9] "Modeling of dielectric materials in FEKO," FEKO Quarterly, March 2005. 